\title{
AKNS Formalism and Exact Solutions of KdV and Modified KdV Equations with Variable-Coefficients
}

\author{
Supratim Das ${ }^{1, a *}$, Dibyendu Ghosh ${ }^{1, b}$ \\ ${ }^{1}$ Department of Applied Mathematics, University of Calcutta, 92 Acharya Prafulla Chandra Road, \\ Kolkata-700009, India \\ asupratimiitkgp@gmail.com, bdghoshbrsc@gmail.com
}

Keywords: Jacobian elliptic function, variable-coefficient KdV and modified KdV equation, AKNS scheme, homogeneous balancing method.

\begin{abstract}
We apply the AKNS hierarchy to derive the generalized KdV equation and the generalized modified $\mathrm{KdV}$ equation with variable-coefficients. We systematically derive new exact solutions for them. The solutions turn out to be expressible in terms of doubly-periodic Jacobian elliptic functions.
\end{abstract}

\section{Introduction}

Some of the most typical natural phenomena that occur in this world are inherently nonlinear and are expected to be guided by an appropriate class of nonlinear evolution equations. Obtaining new exact solutions to nonlinear evolution equations is therefore an important task in the study of nonlinear sciences. In the last few decades, various methods have been used to obtain exact solutions of nonlinear evolution equations, such as the variational iteration method [1], Hirota's bilinear method [2], Backlund transformations [3], direct reduction method [4], inverse scattering method [5], Lax pair formulation [6], sine-cosine method [7], tanh-method [8], homogeneous balancing method [9], to name a few.

Among the simplest nonlinear evolution equations are the Korteweg-de Vries (KdV) equation $[10,11]$

$$
u_{t}+a u u_{x}+b u_{x x x}=0,
$$

where $a$ and $b$ are real constants and the modified $\mathrm{KdV}(\mathrm{mKdV})$ equation $[10,11]$

$$
v_{t}+a_{1} v^{2} v_{x}+b_{1} v_{x x x}=0,
$$

where $a_{1}$ and $b_{1}$ are real constants. These equations appear naturally in a diverse range of physical phenomena $[10,11]$. They are also integrable systems. Their properties are summarized in [12] which gives a comprehensive account of their recursion, Hamiltonian, symplectic and cosymplectic operator, roots of their symmetries and their scaling symmetry.

Different types of exact solutions of the $\mathrm{KdV}$ equation and the $\mathrm{mKdV}$ equation exist which include the solitary wave solutions, positon solutions and $\mathrm{N}$-solition solutions $[10,11,13]$. In the literature, the inverse scattering transform (IST) technique [10] and the AKNS formalism $[14,15,16]$ have been developed to search for a class of soliton solutions pertaining to the nonlinear evolution equations (NLEEs) such as the KdV equation, mKdV equation, nonlinear Schrödinger equation (NLS) and sineGordon equation. The AKNS system is an important physical model as many equations of nonlinear wave propagation in non-uniform medium can be addressed using this model [14, 15]. Gupta [14] derived a nonlinear Schrödinger equation by a simple extension of the Lax integrability criterion [6]. It bears mention that a multi-Hamiltonian structure is a typical feature with $(1+1)$-dimentional Lax integrable system along with infinitely many conservation laws and infinitely many symmetries. However, the presence of time-dependence spectral parameter in non-isospectral equations tends to spoil these characteristics but there are exceptions such as the Ablowitz-Ladik [17], (see also [18]) hierarchy that has two sets of symmetries. There exist many classes of non-isospectral constant-coefficient integrable equations (see [19, 20, 21, 22] for a partial list of such equations) which have been considered in the framework of AKNS scheme. Specifically, in these works the initial value problems 
for the KdV and NLS have been solved and N-soliton solutions have been found. For a history of non-isospectral system dealing with various types of nonlinear evolution equations are refer to Qiao et al [23].

On the other hand, solving for nonlinear evolution equations with variable-coefficients is also important as these equations often model realistic situations in certain case. However, literature on nonlinear evolution equations with variable-coefficients $[24,25,26,27,28,29,30,31,32,33,34$, $35,36,37,38,39]$ is rather limited compared to the constant coefficient counterparts. The AKNS hierarchy of nonlinear evolution equations with variable-coefficients has not been much studied.

Recently, the existence of soliton solutions for the generalized $\mathrm{KdV}$ equation with variable-coefficients

$$
u_{t}+\alpha(t) u u_{x}+\beta(t) u_{x x x}+\{\delta(t)+\gamma(t) x\} u_{x}+\gamma(t) u=0,
$$

where $\alpha(t), \beta(t), \gamma(t)$ and $\delta(t)$ are arbitrary functions of time $t$ has been noted, among others, by Biswas [25, 26], Ma et al [27], Zhang [28], Yu et al [29] and Deng [30] and the solitary wave dynamics were investigated for a specific case of the generalized $\mathrm{mKdV}$ equation with variable-coefficients by Pradhan and Panigrahi [31]

$$
v_{t}+\alpha_{1}(t) v^{2} v_{x}+\beta_{1}(t) v_{x x x}+\left\{\delta_{1}(t)+\gamma_{1}(t) x\right\} v_{x}+\gamma_{1}(t) v=0
$$

where $\alpha_{1}(t), \beta_{1}(t), \gamma_{1}(t)$ and $\delta_{1}(t)$ are arbitrary functions of time $t$.

The aim of this paper is to derive equations (3) and (4) using AKNS hierarchy $[14,15]$ and to obtain the novel exact solutions of these equations which are expressible in terms of doubly-periodic Jacobian elliptic function [5, 40].

This paper is organized as follows. In section 2, we derive the KdV equation and the $\mathrm{mKdV}$ equation with variable-coefficients using AKNS hierarchy. In section 3, an exact solution of the KdV equation with variable-coefficients (3) is obtained. In section 4 , the $\mathrm{mKdV}$ equation with variablecoefficients (4) is being solved to derive an exact solution in a similar procedure applied in section 3. Finally in section 4, we present a summary.

\section{The $\mathrm{KdV}$ equation and the $\mathrm{mKdV}$ equation with variable-coefficients in non-uniform medium using AKNS hierarchy}

The linear eigenvalue problem is given by $[10,14,15]$

$$
L[u] \psi(x, t)=\lambda \psi(x, t),
$$

where $\lambda$ is the eigenvalue and $\psi(x, t)$ evolve with time in a prescribed manner determined by

$$
\partial_{t} \psi(x, t)=A[u] \psi(x, t) .
$$

Let us consider the eigenvalue problem for (5)

$$
\begin{aligned}
& \psi_{x}=M \psi ; \quad \psi=\left[\begin{array}{l}
\psi_{1} \\
\psi_{2}
\end{array}\right] ; \quad M=\left[\begin{array}{cc}
-i \xi & q(x, t) \\
r(x, t) & i \xi
\end{array}\right], \\
& \text { i.e. } \quad\left[\begin{array}{cc}
\frac{\partial}{\partial x} & -q(x, t) \\
r(x, t) & -\frac{\partial}{\partial x}
\end{array}\right]\left[\begin{array}{l}
\psi_{1} \\
\psi_{2}
\end{array}\right]=-i \xi\left[\begin{array}{l}
\psi_{1} \\
\psi_{2}
\end{array}\right],
\end{aligned}
$$

where $q \equiv q(x, t)$ and $r \equiv r(x, t)$ are potentials, $\xi$ is the eigenvalue and the time dependence of the solution is taken according to (6): 


$$
\psi_{t}=N \psi ; \quad N=\left[\begin{array}{cc}
A & B \\
C & D
\end{array}\right] .
$$

The compatibility condition $\psi_{x t}=\psi_{t x}$ reads

$$
N_{x}-M_{t}+[N, M]=\mathbf{0},
$$

where $[N, M]=N M-M N$ and can leads to the following equations

$$
\begin{aligned}
& A_{x}=q C-r B-i \xi_{t}, \\
& B_{x}=-2 i \xi B+q_{t}-2 q A, \\
& C_{x}=2 i \xi C+r_{t}+2 r A, \\
& D_{x}=-A_{x} \text { i.e. } D=-A .
\end{aligned}
$$

One can assume $\xi_{t}$ to be a known polynomial in $\xi$ and set for simplicity $\xi_{t}$ to be linear in $\xi$

$$
\frac{d \xi}{d t}=i(\mu+\eta \xi)
$$

where $\mu$ and $\eta$ are arbitrary functions of time $t$.

Following Gupta [14], expanding $A, B$ and $C$ up to cubic power in $\xi$

$$
\begin{aligned}
& A(x, t)=\sum_{j=0}^{3} A^{(j)}(x, t) \xi^{j}, \\
& B(x, t)=\sum_{j=0}^{3} B^{(j)}(x, t) \xi^{j}, \\
& C(x, t)=\sum_{j=0}^{3} C^{(j)}(x, t) \xi^{j},
\end{aligned}
$$

where $A^{(j)}(x, t), B^{(j)}(x, t)$ and $C^{(j)}(x, t)$ are coefficient functions, we have from equations (11) to (14)

$$
\begin{aligned}
A_{x}^{(j)} & =q C^{(j)}-r B^{(j)}+\mu \delta_{j 0}+\eta \delta_{j 1} ; \quad j=0,1,2,3, \\
B_{x}^{(j)}+2 i B^{(j-1)} & =-2 q A^{(j)} ; \quad j=1,2,3, \\
C_{x}^{(j)}-2 i C^{(j-1)} & =2 r A^{(j)} ; \quad j=1,2,3 .
\end{aligned}
$$

In addition, for $j=0$ equations (12) and (13) lead to the following equations

$$
\begin{aligned}
& q_{t}=2 q A^{(0)}+B_{x}^{(0)}, \\
& r_{t}=-2 r A^{(0)}+C_{x}^{(0)} .
\end{aligned}
$$

To find the $\mathrm{KdV}$ equation and the $\mathrm{mKdV}$ equation with variable-coefficients in a non-uniform medium let us assume

$$
A^{(3)}=f, B^{(3)}=C^{(3)}=0,
$$

where $f$ is an arbitrary function of time $t$. Equations (20) and (21) then imply 


$$
B^{(2)}=i f q, \quad C^{(2)}=i f r .
$$

For $j=2$ equations (19), (20) and (21) can be recast in the form

$$
\begin{aligned}
& A^{(2)}=g, \\
& B^{(1)}=i g q-\frac{1}{2} f q_{x}, \\
& C^{(1)}=i g r+\frac{1}{2} f r_{x},
\end{aligned}
$$

where $g$ is an arbitrary function of time $t$, while for $j=1$ equations (19), (20) and (21) yield

$$
\begin{aligned}
A^{(1)} & =\frac{1}{2} f q r+\eta x+h \\
B^{(0)} & =\frac{i}{2} f r q^{2}+i \eta x q+i h q-\frac{1}{2} g q_{x}-\frac{i}{4} f q_{x x}, \\
C^{(0)} & =\frac{i}{2} f q r^{2}+i \eta x r+i h r+\frac{1}{2} g r_{x}-\frac{i}{4} f r_{x x},
\end{aligned}
$$

where $h$ is an arbitrary function of time $t$.

Turning now to equation (19) we see that $j=0$ gives

$$
A^{(0)}=\frac{1}{2} g q r+\frac{i}{4} f\left(q_{x} r-q r_{x}\right)+\mu x+l,
$$

where $l$ is an arbitrary function of time $t$. On the other hand, on using (30), (31) and (32) equations (22) and (23) can be cast as

$$
\begin{aligned}
& q_{t}=g r q^{2}+2 \mu x q+2 l q+\frac{3 i}{2} f r q q_{x}+i \eta q+i \eta x q_{x}+i h q_{x}-\frac{1}{2} g q_{x x}-\frac{i}{4} f q_{x x x}, \\
& r_{t}=-g q r^{2}-2 \mu x r-2 l r+\frac{3 i}{2} f q r r_{x}+i \eta r+i \eta x r_{x}+i h r_{x}+\frac{1}{2} g r_{x x}-\frac{i}{4} f r_{x x x} .
\end{aligned}
$$

It is interesting to note that equation (33) follows from Gupta's scheme [14] by choosing $\beta, \epsilon, \gamma, \alpha$ and $q^{*}$ suitably and setting $l=h=0$ with constant $f$ and $g$. However, the combined version of equations (33) and (34), which represents a coupled system of independent variables $q$ and $r$, speak of an extended family. Equations (33), (34) are coupled equations of independent variables $q, r$ and $f, g, h$ are functions of time $t$, so in general equation (8) of Gupta [14] can't be reduced to the coupled equations (33), (34). Thus these coupled equations are new and of interest.

With

$$
\begin{aligned}
& g=\mu=l=0, f=-4 i \beta(t)=\frac{2 i}{3} \alpha(t) e^{-i \int \eta d t}, \eta=i \gamma(t), h=i \delta(t), \\
& r(x, t)=e^{i \int \eta d t}, q(x, t)=u(x, t)
\end{aligned}
$$

equations (33) and (34) reduce to the KdV equation with variable-coefficients (3) in a non-uniform medium.

In a similar manner with

$$
\begin{aligned}
& g=\mu=l=0, f=-4 i \beta_{1}(t)=\frac{2 i}{3} \alpha_{1}(t), \eta=i \gamma_{1}(t), h=i \delta_{1}(t), \\
& q(x, t)=r(x, t)=v(x, t),
\end{aligned}
$$

equations (33) and (34) reduce to the $\mathrm{mKdV}$ equation with variable-coefficients (4) in a non-uniform medium. 


\section{Exact solutions of the KdV equation with variable-coefficients}

In this and in the following section we employ the techniques of the so-called homogeneous balancing method [9] to derive exact solutions for the variable-coefficient $\mathrm{KdV}$ equation and its modified partner. To this end, we substitute

$$
u(x, t)=\kappa+\zeta U(X, \tau),
$$

where $\kappa, \zeta, X, \tau$ are the functions of $x, t$ and $U(X, \tau)$ satisfies the equation

$$
U_{\tau}+6 U U_{X}+U_{X X X}=0,
$$

into the $\mathrm{KdV}$ equation with variable-coefficients (3) that results on computing the parameters $\kappa, \zeta, X$, $\tau$ which need term-by-term balancing of the coefficients of $U_{X X X}, U U_{X}, U^{2}, U_{X X}, U, U_{X}$ and $U-$ independent terms to zero. Somewhat involved but straightforward algebra leads to the following relations:

$$
\begin{aligned}
U_{X X X}: & \beta X_{x}^{3}-\tau_{t}=0, \\
U U_{X}: & \alpha \zeta X_{x}-6 \tau_{t}=0, \\
U^{2}: & \zeta_{x}=0 \\
U_{X X}: & \zeta_{x} X_{x}+\zeta X_{x x}=0, \\
U: & \zeta_{t}+\alpha \kappa_{x} \zeta+\gamma \zeta=0, \\
U_{X}: & X_{t}+(\alpha \kappa+\delta+x \gamma) X_{x}=0, \\
U \text { independent }: & \kappa_{t}+\alpha \kappa \kappa_{x}+\beta \kappa_{x x x}+(\delta+x \gamma) \kappa_{x}+\gamma \kappa=0 .
\end{aligned}
$$

Solving this system of equations one can easily obtain

$$
\begin{aligned}
\tau & =\int \frac{\alpha^{3}}{\beta^{2}} d t, \\
X & =\frac{x \alpha}{\beta}+\nu, \\
\zeta & =\frac{6 \alpha}{\beta}, \\
\kappa & =\left[\frac{1}{\beta}(\beta / \alpha)_{t}-\frac{\gamma}{\alpha}\right] x-\frac{\alpha \delta+\beta \nu_{t}}{\alpha^{2}}
\end{aligned}
$$

in the presence of the following two conditions

$$
\alpha^{2}\left(\alpha_{t} / \alpha^{3}\right)_{t}+\alpha(\gamma / \alpha)_{t}+\gamma^{2}+\frac{3 \alpha_{t} \beta_{t}-\alpha \beta_{t t}}{\alpha \beta}=0
$$

and

$$
\frac{\nu_{t t}}{\nu_{t}}+\frac{2 \beta_{t}}{\beta}-\frac{3 \alpha_{t}}{\alpha}+\frac{\alpha^{2}}{\beta \nu_{t}}(\delta / \alpha)_{t}+\frac{\alpha \gamma \delta}{\beta \nu_{t}}=0,
$$

where $\nu$ is an arbitrary function of time $t$. We furnish a partial list of specific values of $\alpha, \beta, \gamma, \delta, \nu$ which satisfies the conditions (50) and (51) are 
- $\quad \alpha=e^{m t}, \beta=e^{n t},(m, n \in \mathbb{R}), \gamma=\frac{m}{2}-\Lambda \tan \Lambda t$,

$\Lambda=\left(3 m n-n^{2}-\frac{9}{4} m^{2}\right)^{1 / 2}, \delta$ is arbitrary,

$\nu=\int\left[e^{(3 m-2 n) t} \int\left(m \delta-\gamma \delta-\delta_{t}\right) e^{(n-2 m) t} d t\right] d t$.

- $\quad \alpha=$ constant, $\beta=e^{n t},(n \in \mathbb{R}), \gamma=n \operatorname{coth} n t, \delta$ is arbitrary,

$\nu=-\alpha \int\left[e^{-2 n t} \int\left(\gamma \delta+\delta_{t}\right) e^{n t} d t\right] d t$

- $\quad \alpha=e^{m t},(m \in \mathbb{R}), \beta=$ constant, $\gamma=\frac{m}{2}+\frac{3 m}{2} \operatorname{coth} \frac{3}{2} m t$,

$\delta$ is arbitrary, $\nu=\frac{1}{\beta} \int\left[e^{3 m t} \int\left(m \delta-\gamma \delta-\delta_{t}\right) e^{-2 m t} d t\right] d t$.

- $\quad \alpha=\beta=t^{n},(n \in \mathbb{R}), \gamma=0, \delta$ is arbitrary,

$\nu=\int\left[t^{n} \int\left(n \delta-t \delta_{t}\right) t^{-n-1} d t\right] d t$.

- $\quad \alpha=t^{n}, \beta=t^{1+2 n},(n \in \mathbb{R}), \gamma=0, \delta$ is arbitrary,

$\nu=\int\left[t^{-n-2} \int\left(n \delta-t \delta_{t}\right) d t\right] d t$.

Equation (38) admits a cnoidal solution [10]

$$
U(X, \tau)=\frac{2 c^{2} k^{2}}{2 k^{2}-1} \mathrm{cn}^{2}\left[\frac{c}{\sqrt{2 k^{2}-1}}\left(X-4 c^{2} \tau\right), k\right]
$$

where $\operatorname{cn}(\theta, k)$ is the Jacobi elliptic cosine function, $k$ is the modulus of the elliptic function and $c$ is an arbitrary constant.

The final form of the solution of equation (3) is

$$
u(x, t)=\kappa+\frac{12 \alpha c^{2} k^{2}}{\beta\left(2 k^{2}-1\right)} \operatorname{cn}^{2}\left[\frac{c}{\sqrt{2 k^{2}-1}}\left(X-4 c^{2} \tau\right), k\right],
$$

where $\tau, X$ and $\kappa$ are given in (46), (47) and (49) respectively.

In the limit case $k \rightarrow 1$, the above reduces to the standard $\operatorname{sech}^{2}$-profile :

$$
u(x, t)=\kappa+\frac{12 \alpha c^{2}}{\beta} \operatorname{sech}^{2}\left[c\left(X-4 c^{2} \tau\right)\right]
$$

\section{Exact solutions of the modified $\mathrm{KdV}$ equation with variable-coefficients}

To obtain exact solutions of the equation (4) we consider the following ansatz for $v(x, t)$

$$
v(x, t)=\kappa+\zeta V(X, \tau),
$$

where $\kappa, \zeta, X, \tau$ are the functions of $x, t$ and $V(X, \tau)$ satisfies the equation

$$
V_{\tau}+6 V^{2} V_{X}+V_{X X X}=0 .
$$

Substituting (60) into equation (4) and balancing the coefficients of $V_{X X X}, V V_{X}, V^{2} V_{X}, V^{3}, V^{2}$, $V_{X X}, V_{X}, V, V$-independent terms to zero give the following set of equations 


$$
\begin{aligned}
V_{X X X}: & \tau_{t}-\beta_{1} X_{x}^{3}=0, \\
V V_{X}: & \kappa=0 \\
V^{2} V_{X}: & 6 \tau_{t}-\alpha_{1} \zeta^{2} X_{x}=0 \\
V^{3}: & \zeta_{x}=0 \\
V^{2}: & \kappa_{x} \zeta+2 \kappa \zeta_{x}=0 \\
V_{X X}: & \beta_{1} \zeta X_{x} X_{x x}=0 \\
V_{X}: & X_{t}+\left(\delta_{1}+x \gamma_{1}\right) X_{x}+\beta_{1} X_{x x x}=0, \\
V: & \zeta_{t}+\gamma_{1} \zeta=0 \\
V-\text { independent }: & \kappa_{t}+\alpha_{1} \kappa^{2} \kappa_{x}+\beta_{1} \kappa_{x x x}+\left(\delta_{1}+x \gamma_{1}\right) \kappa_{x}+\gamma_{1} \kappa=0 .
\end{aligned}
$$

The non-trivial solution of this system of equations can be obtained as

$$
\begin{aligned}
X & =-\int\left[\left(\delta_{1}+x \gamma_{1}\right) \sqrt{\frac{\alpha_{1}}{6 \beta_{1}}} e^{-\int \gamma_{1} d t}\right] d t \\
\tau & =\int\left(\frac{\alpha_{1}}{6 \beta_{1}^{\frac{1}{3}}}\right)^{\frac{3}{2}} e^{-3 \int \gamma_{1} d t} d t \\
\zeta & =e^{-\int \gamma_{1} d t} \\
\kappa & =0 .
\end{aligned}
$$

Equation (61) possesses a well-known solution [10]

$$
V(X, \tau)=k \operatorname{cn}\left[X-\left(1-2 k^{2}\right) \tau, k\right]
$$

which provides from (60) the exact solution of the variable-coefficient $\mathrm{mKdV}$ equation (4) namely

$$
v(x, t)=k e^{-\int \gamma_{1} d t} \operatorname{cn}\left[X-\left(1-2 k^{2}\right) \tau, k\right],
$$

where $X, \tau$ are given in (71) and (72).

In the limit case $k \rightarrow 1$, solution (76) goes over to

$$
v(x, t)=e^{-\int \gamma_{1} d t} \operatorname{sech}(X+\tau) .
$$

\section{Summary}

Observing that the most important key to deriving the variable-coefficient integrable equations is to extend the AKNS formalism from the isospectral problem to a non-isospectral one (involving explicitly the time-dependent parameter $\xi$ ), we have shown that specific coupled versions of the variablecoefficient $\mathrm{KdV}$ equation and its modified partner exist which are reducible to the AKNS formalism. We have then proposed a homogeneous balancing method to obtain a new classes of general exact solutions expressible in terms of doubly-periodic Jacobian elliptic functions.

\section{Acknowledgment}

We thank Prof. Bijan Kumar Bagchi for his valuable guidance. SD thanks CSIR, India for providing a Senior Research Fellowship. 


\section{References}

[1] J. H. He, Variational iteration method-a kind of non-linear analytical technique: some examples, Inter. J. Nonlin. Mech. 34(4) (1999) 699-708.

[2] R. Hirota, Exact solution of the Korteweg - de Vries equation for multiple collisions of solitons, Phys. Rev. Lett. 27(18) (1971) 1192.

[3] M. R. Miura, Backlund Transformation, Springer-Verlag, Berlin, 1978.

[4] Z. Yan, H. Zhang, Nonlinear wave agenda of similar reduction with damping term, Physics Journals. 49(11) (2000) 2113-2117.

[5] M. J. Ablowitz, P. A. Clarkson, Soliton, nonlinear evolution equation and inverse scattering, Cambridge University press, New York, 1991.

[6] P. D. Lax, Integrals of nonlinear equations of evolution and solitary waves, Comm. Pure. Appl. Math. 21(5) (1968) 467-490.

[7] C. Yan, A simple transformation for nonlinear waves, Phys. Lett. A. 224(1) (1996) 77-84.

[8] S. Yu et al., Solitary wave solutions to approximate fully nonlinear double sine-Gordon equation, International Journal of Nonlinear Science. 3(3) (2007) 163-169.

[9] J. F. Zhang, Homogeneous balance method and chaotic and fractal solutions for the NizhnikNovikov-Veselov equation, Phys. Lett. A. 313(5) (2003) 401-407.

[10] P. G. Drazin, R. S. Johnson, Solitons: An Introduction, Cambridge University press, London, 1983.

[11] M. Lakshmanan, S. Rajasekar, Nonlinear dynamics : Integrability, Chaos and Patterns, Advanced Texts in Physics, Springer-Verlag, Berlin, 2003

[12] J. P. Wang, A list of 1+ 1 dimensional integrable equations and their properties, Journal of Nonlinear Math. Phys. 9 (2002) 213-233.

[13] V.B. Matveev, Generalized Wronskian formula for solutions of the KdV equations: first applications, Phys. Lett. A. 166(3-4) (1992) 205-208.

[14] M.R. Gupta, Exact inverse scattering solution of a non-linear evolution equation in a non-uniform medium, Phys. Lett. A. 72(6) (1979) 420-422.

[15] M.J. Ablowitz et al., The Inverse Scattering Transform-Fourier Analysis for Nonlinear Problems, Stud. Appl. Math. 53(4) (1974) 249-315.

[16] M.J. Ablowitz et al., Nonlinear-evolution equations of physical significance, Phys. Rev. Lett. 31(2) (1973) 125.

[17] M.J. Ablowitz, J.F. Ladik, Nonlinear differential- difference equations, Journal of Mathematical Physics. 16(3) (1975) 598-603.

[18] W. Hua, D.J. Zhang, Strong Symmetries of Non-Isospectral Ablowitz-Ladik Equations, Chin. Phys. Lett. 28(2) (2011) 020203

[19] H.H. Chen, C.S. Liu, Solitons in nonuniform media, Phys. Rev. Lett. 37(11) (1976) 693. 
[20] R. Hirota, J. Satsuma, N-soliton solution of the K-dV equation with loss and nonuniformity terms, Journal of the Physical Society of Japan. 41 (1976) 2141

[21] W.L. Chan. L. Kam-Shun, Nonpropagating solitons of the variable coefficient and nonisospectral Korteweg-de Vries equation, J. Math. Phys. 30(11) (1989) 2521-2526.

[22] J.B. Bi, Y.P. Sun, D.Y. Chen, Soliton Solutions for Nonisospectral AKNS Equation by Hirota's Method, Communications in Theoretical Physics. 45(3) (2006) 398.

[23] Z. Qiao, C. Cao, W. Strampp, Category of nonlinear evolution equtions, algebraic structure, and $r$-matrix, Unpublished paper.

[24] Z.T. Fu et al., New exact solutions to KdV equations with variable coefficients or forcing, Applied Mathematics and Mechanics. 25(1) (2004) 73-79.

[25] A. Biswas, Solitary wave solution for KdV equation with power-law nonlinearity and timedependent coefficients, Nonlinear Dynamics. 58(1-2) (2009) 345-348.

[26] A. Biswas, Solitary wave solution for the generalized KdV equation with time-dependent damping and dispersion, Communications in Nonlinear Science and Numerical Simulation. 14(9) (2009) 3503-3506.

[27] H. Ma, A. Deng, Y. Wang, Exact solution of a KdV equation with variable coefficients, Computers and Mathematics with Applications. 61(8) (2011) 2278-2280.

[28] S. Zhang, Application of Exp-function method to a $\mathrm{KdV}$ equation with variable coefficients, Phys. Lett. A. 365(5) (2007) 448-453.

[29] S.M. Yu, L.X. Tian, Generalized soliton solutions to generalized KdV equation with variable coefficients by Exp-function method, Journal of Physics: Conference Series. 96(1) (2008) 012022.

[30] S.F. Deng, Exact solutions for a nonisospectral and variable-coefficient kdv equation, Communications in Theoretical Physics. 43(6) (2005) 961.

[31] K. Pradhan, P. K. Panigrahi, Parametrically controlling solitary wave dynamics in the modified Korteweg-de Vries equation, Journal of Physics A: Mathematical and General. 39(20) (2006) L343.

[32] J. Li et al., Symbolic computation on integrable properties of a variable-coefficient Korteweg-de Vries equation from arterial mechanics and Bose-Einstein condensates, Physica Scripta. 75(3) (2007) 278.

[33] J. Li et al., Lax pair, Bäcklund transformation and N-soliton-like solution for a variablecoefficient Gardner equation from nonlinear lattice, plasma physics and ocean dynamics with symbolic computation, Journal of Mathematical Analysis and Applications. 336(2) (2007) 14431455.

[34] X.L. Gai et al., On a variable-coefficient Korteweg-de Vries model in fluid-filled elastic tubes, Journal of Physics A: Mathematical and Theoretical. 43(45) (2010) 455205.

[35] Y. Jiang et al., Soliton solutions for a variable-coefficient Korteweg? de Vries equation in fluids and plasmas, Physica Scripta. 82(5) (2010) 055008.

[36] A.G. Johnpillai, C.M. Khalique, Group analysis of KdV equation with time dependent coefficients, Applied Mathematics and Computation. 216(12) (2010) 3761-3771. 
[37] C.A.G. Sierra, On a KdV equation with higher-order nonlinearity: Traveling wave solutions, Journal of computational and applied mathematics. 235(17) (2011) 5330-5332.

[38] Y. Qin et al., Bell polynomial approach and N-soliton solutions for a coupled KdV-mKdV system, Communications in Theoretical Physics. 58(1) (2012) 73.

[39] Y. Jiang et al., Soliton solutions and integrability for the generalized variable-coefficient extended Korteweg-de Vries equation in fluids, Applied Mathematics Letters. 26(4) (2013) 402-407.

[40] Y.F. Xiao, H.L. Xue, The new multi-order exact solutions of some nonlinear evolution equations, Journal of Atomic and Molecular Sciences. 3 (2012) 136-151. 